科 学 通 报

\title{
黄腐酸与大鼠肝细胞微粒体的相互作用 *
}

\author{
张法浩李蓉 许善锦王益 \\ (北京医科大学药学院, 北京 100083)
}

\section{关钺词费席酸、微粒体、自由基}

我们已报道过黄腐酸 (Fulvic acid, FA) 可引发大鼠肝细胞产生活性氧自由基 ${ }^{[1]}$, 并导致生 物体的氧化性损伤 ${ }^{[2]}$. 但是, 作为一种外源性物质, 黄腐酸在细胞内怎样转化, 通过何种机制 引发肝细胞产生内源性活性氧物质, 尚不清楚. 一般认为, 外源性物质多是通过肝细胞内氧 化 - 还原酶体系进行生物转化, 进而产生活性氧物质. 而此类酶体系多存在于线粒体或微粒 体中. 本文以大鼠肝细胞线粒体和微粒体为研究对象, 研究了黄腐酸与微粒体、线粒体的相 互作用过程, 并探讨了产生活性氧的可能机制.

\section{1 材 料 和 方 法}

\section{1 实验材料}

还原性辅酶 II(NADPH) 购自 Sigma 公司, 二硝基四氮唑蓝 (NBT) 购自华美公司, 超氧化 物歧化酶 (SOD) 为中国科学院生物物理研究所产品, 其它均为国产分析纯试剂. 黄腐酸 (FA) 由中国科学院生态环境研究中心提供, 实验用 Wistar 大鼠由北京医科大学实验动物部 提供.

\section{2 实验方法}

(1) 肝细胞线粒体和微粒体的分离制备. 选择 180-250g 雄性 Wistar 大鼠经连续 $3 \mathrm{~d}$ 腹 腔注射苯巴比妥钠 $(75 \mathrm{mg} / \mathrm{kg} \cdot \mathrm{d})$, 禁食 $16 \mathrm{~h}$ 后, 断头处死. 取出肝脏, 参照文献[3] 方法分别制 备线粒体和微粒体, 并按 Lowry 法 ${ }^{[4]}$ 测定制备的线粒体和微粒体蛋白含量.

（2）氧自由基检测. 在磷酸缓冲溶液 $(50 \mathrm{mmol} / \mathrm{L}, \mathrm{pH} 7.4)$ 中分别加人微粒体或线粒体 $(1.0 \mathrm{mg}$ 蛋白 $/ \mathrm{mL}), 37^{\circ} \mathrm{C}$ 温育 $5 \mathrm{~min}$. 加人 $\mathrm{NADPH}(1.0 \mathrm{mmol} / \mathrm{L})$ 后用 $\mathrm{NBT}(50 \mu \mathrm{mol} / \mathrm{L})$ 还原光 度法测定超氧自由基 $\left(\cdot \mathrm{O}_{2}^{-}\right)$.

(3) 紫外差谱. 将微粒体 $(1.5 \mathrm{mg}$ 蛋白 $/ \mathrm{mL})$ 加人缓冲溶液 (50mmol/ $\mathrm{L} \mathrm{Na} \mathrm{NPO}_{4}, 0.10$ $\mathrm{mol} / \mathrm{L} \mathrm{NaCl}, \mathrm{pH}$ 7.4) 中, 制成微粒体悬浮液. 用 UV-260 分光光度计分别记录加人 $\mathrm{FA}$ 前后 和加人 NADPH 前后的紫外吸收差谱.

(4) ESR 谱. 分别在有氧和无氧 (通 $\mathrm{N}_{2}$ 密闭) 条件下使微粒体 (1.0mg 蛋白 $/ \mathrm{mL}$ ) 与 $\mathrm{FA}$ $\left(25 \times 10^{-6}\right)$ 温育 $\left(37^{\circ} \mathrm{C}\right) 2 \mathrm{~min}$, 加人 NADPH $(1.0 \mathrm{mmol} / \mathrm{L})$ 后用 BRUKER ESP-300 型波谱仪记 录 ESR 谱.

1994-03-12 收稿, 1994-06-06 收修改稿.

*国家“八五”科技攻关资助项目. 


\section{2 实 验 结 果}

\section{1 活性舞自由基的产生}

图 1 是微粒体或线粒体与 FA 作用体系中的 NBT 还原产物量 $\left(A_{s 60}\right)$ 的时间变化曲线. 由 图 1 可见, 反应体系中的 NBT 可被还原, 并且在加人超氧化物歧化酶 (SOD) 后, 可明显地抑 制 NBT 的还原. 提示 NBT 是被反应体系中产生的超氧阴离子 $\left(\cdot \mathrm{O}_{2}^{-}\right)$所还原. 说明微粒体 和线粒体在 FA 的作用下, 有活性氧物质产生, 同时也证明微粒体的活性氧产生量远远高于线 粒体.

表 1 反应体系中活性篻相对含量 ${ }^{a)}$

\begin{tabular}{lll}
\hline \multicolumn{1}{c}{ 反应体系 } & \multicolumn{1}{c}{ 微粒体 } & 线粒体 \\
\hline 完全体系 $^{\mathrm{b})}$ & $100 \pm 11.4$ & $15.7 \pm 5.6$ \\
$+\mathrm{SOD}(50 \mu \mathrm{g} / \mathrm{mL})$ & $37.8 \pm 18.0$ & $6.8 \pm 3.5$ \\
- 微粒体 $($ 线粒体 $)$ & $8.5 \pm 8.0$ & $4.0 \pm 4.0$ \\
- NADPH & $8.2 \pm 8.0$ & $8.0 \pm 4.5$ \\
-FA & $14.8 \pm 5.1$ & $5.6 \pm 3.2$ \\
+ 咪唑 $(1.0 \mathrm{mmol} / \mathrm{L})$ & $56.5 \pm 8.5$ & $9.8 \pm 3.0$ \\
\hline
\end{tabular}

a) $n=4$, 数据为 $X \pm \mathrm{Sx}$. b) 完全体系中含有 $1.0 \mathrm{mg}$ 蛋白 $/ \mathrm{mL}$ 微 粒体 (或线粒体)， $1.0 \mathrm{mmol} / \mathrm{L} \mathrm{NADPH}, 25 \times 10^{-6} \mathrm{FA}$. 其它反 应体系均在完全体系的基础上加人或除去一种物质.

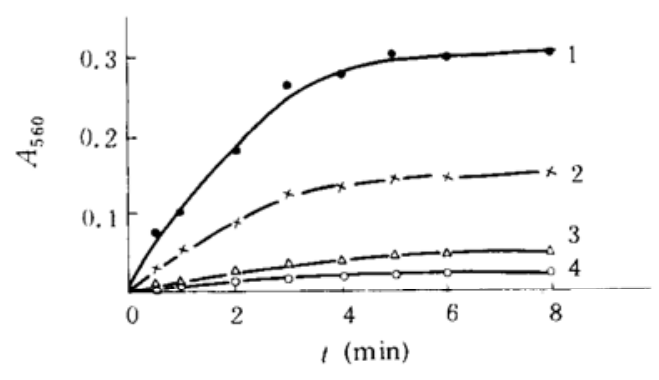

图 1 NBT 还原产物量与时间关系曲线 1 为微粒体 $(1.0 \mathrm{mg} / \mathrm{mL})+\mathrm{NADPH}(1.0 \mathrm{mmol} / \mathrm{L})+\mathrm{FA}$ $\left(25 \times 10^{-6}\right), 2$ 为微粒体 + NADPH + FA + SOD (50 $\mu \mathrm{g} / \mathrm{mL}), 3$ 为线粒体 $(1.0 \mathrm{mg} / \mathrm{mL})+\mathrm{NADPH}(1.0 \mathrm{mmol} / \mathrm{L})$ $+\mathrm{FA}\left(25 \times 10^{-6}\right), 4$ 为线粒 体 + NADPH + FA + SOD $(50 \mu \mathrm{g} / \mathrm{mL})$

另外, 表 1 列出了各反应体系用 NBT 还原光度法测定的活性氧相对含量的结果 (按 FA 和 NADPH 同时作用于微粒体所产生的活性氧为 100 计算).

由实验结果可见, 只有 FA, NADPH 和微粒体同时存在的反应体系(完全体系)才可产生 大量的活性氧物质, 除去 FA, NADPH 和微粒体 3 种组分的任 1 种只产生很少或可忽略量的 活性氧. 另外 2 个值得注意的结果是, 线粒体产生活性氧的能力非常显著地低于微粒体, 提示 FA 引发肝细胞产生活性氧的部位主要在细胞内的微粒体; 咪唑明显地抑制微粒体产生活性 氧.

不同浓度的 FA 及不同来源的 FA(大骨节病区和非病区的 FA) 在 NADPH 存在下与微 粒体作用产生活性氧的相对量也有明显差异 (表 2), 这一结果与我们细胞实验的结果 ${ }^{[1]}$ 是一 致的.

表 2 FA 浓度和来源与微粒体产生活性氧含量的关系 ${ }^{a)}$

\begin{tabular}{cccccc}
\hline FA 浓度 $\left(\times 10^{-6}\right)$ & 5.0 & 15 & 25 & 35 & 50 \\
\hline 病区 FA & $14.5 \pm 3.7$ & $57.0 \pm 6.4$ & $100 \pm 11.4$ & $117 \pm 13.5$ & $123 \pm 11.2$ \\
非病区 FA & $13.6 \pm 4.0$ & $24.5 \pm 9.5$ & $53.0 \pm 12.7$ & $59.8 \pm 10.3$ & $62.9 \pm 3.2$ \\
\hline
\end{tabular}

a) $n=4$, 数据为 $X \pm S x$.

\subsection{FA 与细胞色衰 P-450 的作用}

已知咪唑作为细胞色素 P-450 的中心铁离子的 1 个结合配体, 可抑制细胞色素 P-450 的 电子传递功能 ${ }^{[5]}$. 本文的咪唑可抑制微粒体产生活性氧的结果提示大量存在于微粒体中的细 
胞色素 P-450 可能参与了 FA 的生物转化. 由 FA 与微粒体作用后的紫外差谱 (图 2) 可见, 在 $\approx 380 \mathrm{~nm}$ 有一峰, 在 $\approx 420 \mathrm{~nm}$ 有一谷, 提示 FA 与微粒体中的细胞色素 P-450 分子发生了 结合作用 ${ }^{[}$, 并与加人的 FA 浓度呈正相关.

另外, 在 FA 与微粒体体系中加人 NADPH 并通人 $\mathrm{CO}$ 气体可见 $450 \mathrm{~nm}$ 处出现还原态 P450 与 $C O$ 结合的特征吸收峰 (图 3), 而末加 FA 的体系中未见 $450 \mathrm{~nm}$ 处出现强吸收峰. 提 示与 FA 结合后的 P-450 更易被 NADPH 还原.

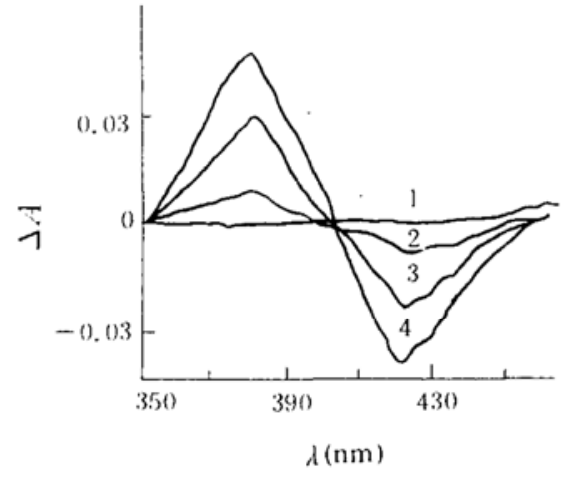

图 2 FA 与微粒体作用的差谱 1 为基线, 2 为加人 $15 \times 10^{-6} \mathrm{FA}, 3$ 为加人 $25 \times 10^{-6} \mathrm{FA}$, 4 为加人 $50 \times 10^{-6} \mathrm{FA}$
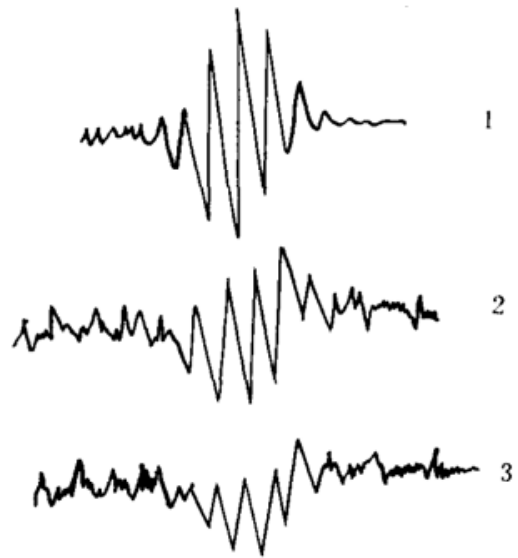

图 4 FA、微粒体及 NADPH 反应体系的 ESR 波谱 1 为无氧条件下反应 $5 \mathrm{~min}, 2$ 为有氧条件下反应 $2 \mathrm{~min}, 3$ 为 有氧条件下反应 $5 \mathrm{~min}$



图 3 NADPH 与微粒体作用的差谱(测定前通人 $\mathrm{CO})$

1 为基线, 2 为微粒体中加人 NADPH, 3 为微粒体中加 人 FA 和 NADPH

\subsection{ESR 谱与半酯自由基}

由 FA 与微粒体及 NADPH 反应体系所 测得的 ESR 谱(图 4)可见, 有半醌自由基信 号出现, 并在无氧条件下可保持信号较长时 间 $(10 \mathrm{~min})$ 恒定, 但在有氧条件下, 信号强度 较弱并随时间降低. 说明在 NADPH 存在 下, 与微粒体中 P-450 结合的 FA 可被还原活 化为半醌自由基, 活性较强的半醌自由基又 可进一步与分子氧作用而呈不稳定状态.

\section{3 讨 论}

微粒体和线粒体是已知的肝细胞内可发 生氧化还原作用的 2 个主要亚细胞组分, 在 外源性物质的分解、生物转化过程中起重要 作用. 本文的结果说明, 黄腐酸 (FA) 主要与

微粒体作用而产生活性氧自由基, 咪唑能抑制活性氧产生的结果提示了细胞色素 P-450 参与 了 FA 的转化的可能性. 进一步的实验证明, FA 与微粒体中细胞色素 P-450 发生了结合, 并 使低自旋态的 P-450 转化为易被 NADPH 还原的高自旋态. 半醌自由基的产生可能是还原态 的 P-450 分子将单电子转移给已结合在其分子上的 FA, 使含醌式结构的 FA 还原的结果. 生 


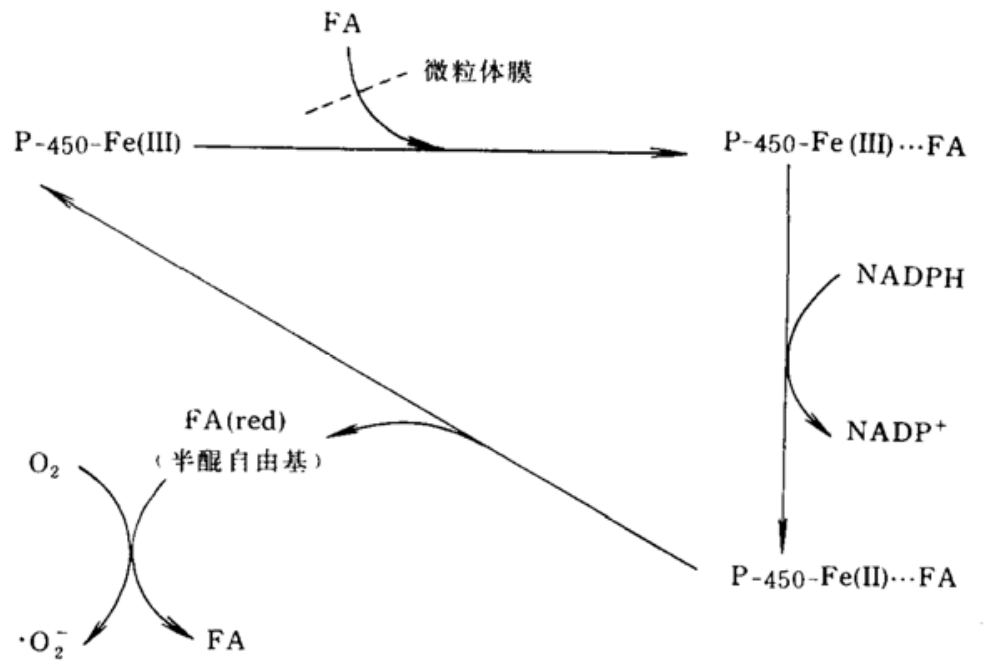

图 5 FA 与 P-450相互作用及产生活性氧的过程

成的半醌自由基在无氧条件比较稳定而在有氧条件下不稳定的结果说明活性较强的半醌自由 基可与分子氧发生反应, 而活性氧自由基可能就产生于半醌自由基将单电子再转移给分子氧 的过程中.

综上所述, 黄腐酸与微粒体相互作用并产生活性氧自由基的可能机制如图 5.

\section{参考文輬}

[1] 张法浩等, 科学通报, 1993, 38(13): 1235.

[2] Zhang, F. H. et al., J. Environ. Sci. (China), 1991, 3(4): 48.

[3] Won, W. D. et al., Appl. Environ. Microbiol., 1976, 31: 576.

[4] Lowry, O. H. et al., J. Biol. Chem., 1951, 193: 265.

[ 5] Testa, B., Jenner, P., Drug. Met. Rev., 1981, 12:1.

[6] Gibson, G. G., Tamburine, P. P., Xenobiotica, 1984, 14: 27. 